FERMIIAB-Conf-82/69-EXP

7550.537

7184.326

DIMUON PRODUCTION IN HADRONIC INTERACTIONS*

B. $\operatorname{Cox}$

October 1982

* Rapporteur Talk XXI International Conference on High Energy Physics, Paris, France, July 26-31, 1982. 


\section{DIMUON PRODUCTION IN HADRONIC INTERACTIONS}

B. Cox

Fermi National Accelerator Laboratory

Rapporteur Talk

XXI International Conference on High Energy Physics

Paris, France

\section{Abstract}

This report summarizes the new data submitted to the XXI International Conference on High Energy Physics on high mass dimuon production in $\pi^{-} N$ and $\bar{p} N$ interactions by Fermilab Experiment E-537, CERN Experiment NA3 and Fermilab Experiment E-326. Successes and failures of the Drell-Yan model and low order QCD are reviewed. New results on the production of the $J / \psi$ from E-537, NA3 and CERN experiment WAIl are also reported. 
Introduction - Experiments which measure the production of high mass (M> $4 \mathrm{GeV} / \mathrm{c}^{2}$ ) dilepton pairs present opportunities to confront the constituent picture of hadrons which has evolved in the last decade. Comparison of the dilepton data with the predictions of the Orell-Yan mode ${ }^{1}$ (zeroth order QCD) and the Compton and annihilation processes ${ }^{2}$ (first order QCD) allow the quark and gluon structure of the hadrons to be determined. Studies of the production of lepton pairs in ón collisions provide an opportunity to test the Drell-Yan and QCD picture of hadronic production of lepton pairs using the nucleon structure functions determined in deep inelastic lepton scattering experiments. Measurements of high mass lepton pair production in $\pi N$ interactions make it possible to measure pion structure functions which cannot be determined in deep inelastic lepton scattering experiments. New data has been presented at this conference on the production of high mass lepton pairs in both $\overline{p N}$ and $\pi^{-} \mathrm{N}$ interactions. ${ }^{3,4,5}$

In addition to the new data on the high mass lepton pair production new data has been reported at this conference on the production of $J / \psi(3.1)$ in $\bar{p} N$ and $\pi^{-} N$

interactions. ${ }^{-6}$ In the same manner that high mass pair production sheds light on hadronic quark structure functions, the measurement of direct $\mathrm{J} / \psi$ production has been used to determine gluon structure functions." Finally the observation of multiple $\psi$ production has been reported. ${ }^{8}$ The production of $d i-\psi$ events may be an indication of rare processes such as $\vec{B} B$ production.

High Mass Muon Pair Production - The confrontation of Drell-Yan and first order QCD with the high mass muon pair production data has had both successes and failures to date. The inability of the naive Drell-Yan model to predict the absolute level of the cross sections for $\mu$ pair production has been quantified as the ' $K$ factor' for $\pi N$ and $\bar{p} N$ reactions. This quantity is defined as the ratio of the measured cross section to the Drell-Yan prediction (using some choice of hadronic structure functions such as the CDHS nucleon structure functions). The preliminary new results (denoted by the astericks) from E-537 for $K$ are shown in Table 1 along with the results of previous experiments.

Table 1: Experimental K Factors ( $K=\frac{d \sigma}{d M d X_{F}}$ data/ $\frac{d \sigma}{d M d X_{F}}$ Drell-Yan)

\begin{tabular}{|c|c|c|c|}
\hline$P_{\text {Lab }}$ & Beam/Target & K & Experiment \\
\hline $\begin{array}{r}* \quad 125 \\
150 \\
\end{array}$ & $\begin{array}{l}\overline{\mathrm{e}}_{\mathrm{N}} \\
\mathrm{p} \mathrm{N} \\
\end{array}$ & $\begin{array}{l}2.25 \pm 0.45 \\
2.3 \pm 0.4\end{array}$ & $\begin{array}{l}\text { Fermilab E-537 } \\
\text { CERN NA3 }\end{array}$ \\
\hline $\begin{array}{l}39.5 \\
\star \quad 125 \\
150,200 \\
150 \\
150 \\
150,175 \\
\end{array}$ & $\begin{array}{l}\pi^{ \pm} N \\
\pi^{-N} N \\
\pi^{-N} \\
\pi^{-}-\mathrm{P} \\
\pi^{-} \mathrm{P}\end{array}$ & $\begin{array}{l}2.6 \pm 0.5 \\
2.5 \pm 0.5 \\
2.2 \pm 0.3 \\
2.4 \pm 0.4 \\
2.4 \pm 0.4 \\
2.8\end{array}$ & $\begin{array}{l}\text { CERN BCE (Omega) } \\
\text { Fermi lab } \\
\text { CERN NA3 } \\
\text { CERN NA3 } \\
\text { CERN NA3 } \\
\text { CERN WAIl }\end{array}$ \\
\hline $\begin{array}{r}150 \\
400 \\
400 \\
2115 \quad(\sqrt{5}=63) \\
\end{array}$ & $\begin{array}{l}\mathrm{pN} \\
\mathrm{pN} \\
\mathrm{pN} \\
\mathrm{pp}\end{array}$ & $\begin{array}{l}2.2 \pm 0.4 \\
1.7 \\
1.6 \pm 0.3 \\
1.6\end{array}$ & $\begin{array}{l}\text { CERN NA3 } \\
\text { Fermilab E-288 } \\
\text { Fermi lab E-439 } \\
\text { CERN R-209 }\end{array}$ \\
\hline
\end{tabular}


The data given in Table 1 for $\pi N$ reaction are consistent wi th one another but the oN data show three experiments with $K$ factors of approximately 1.6 to 1.7 and one experiment, NA3 with a nucleon $K$ factor of 2.2. The new $\bar{p} N$ data shown by $E-537$ is the only other experiment besides the NA3 experiment to obtain a K factor greater than 2 for the nucleon or antinucleon reactions. Other than the problem with the nucleon $k$ factors the data seem consistent with a $K$ factor relatively independent of beam type and in general greater than two. No experimental evidence has yet been presented that would suggest that the $K$ factor is a function of any kinematic variable.

Using Proton West enriched antiproton beam (obtained from $\bar{\Lambda} \rightarrow \bar{p}^{+}$decays) Fermilab Experiment E-537 recently completed the highest statistics measurement of $\bar{p}^{N} \rightarrow \mu^{+} \mu^{-}$ to date.
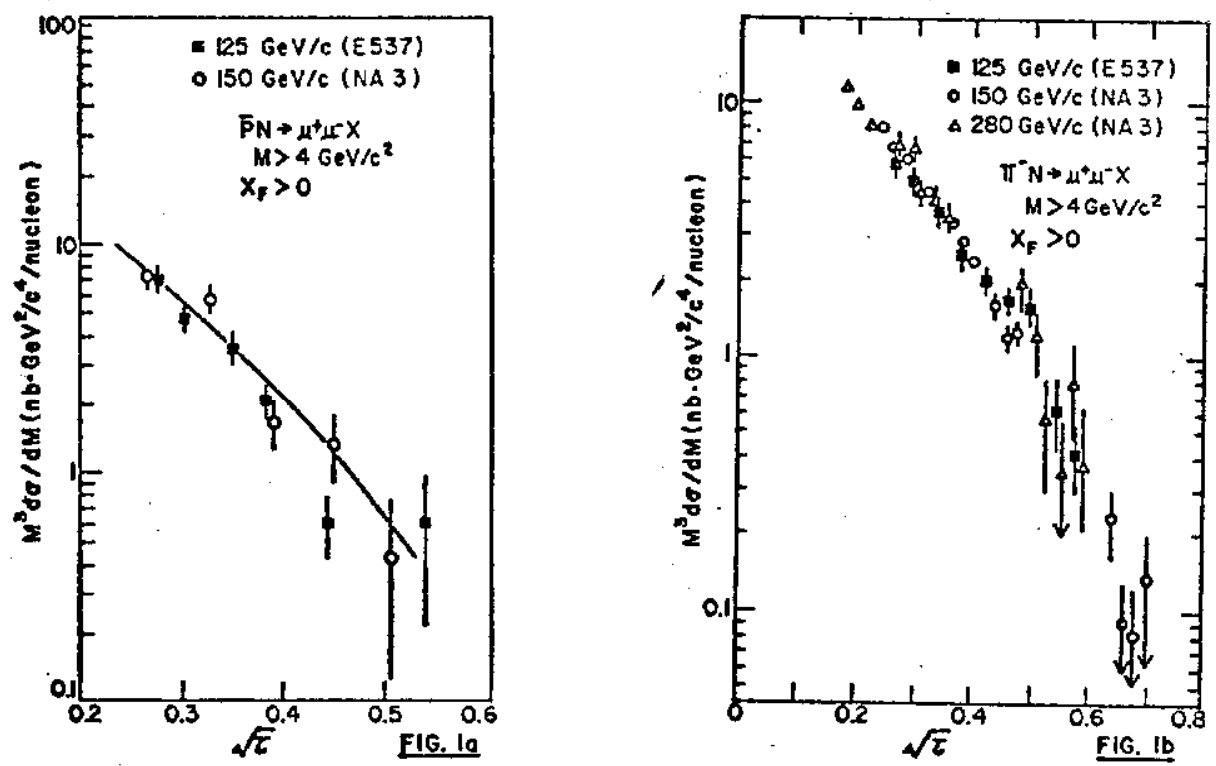

The scaling cross section, $M 3 \mathrm{dd} / \mathrm{dM}$, determined by the E-537 experimenters is shown in Fig. la along with the higher energy NA3 $\bar{p} N$ data. The solid line is 2.3 times the simple Drell-Yan calculation of this cross section obtained by using the CDHS structure functions. ${ }^{2}$ Figure lb shows new data from $E-537$ for $\pi^{-} \mathrm{N} \rightarrow \mu^{+} \mu^{-}$along with older data from NA3 at other energies. These figures indicate two of the successes of the simple Drell-Yan model (prediction of the shape of $M^{3} \mathrm{d \sigma} / \mathrm{d} M$ and "the scaling property of this cross section) and one of the failures (inability to predict the absolute level of the cross section).

A third success of simple Drell-Yan has been the prediction of the shape of the $X_{F}$ distribution for dimuon production. In particular, as seen from the E-537 data shown in Fig. 2, shape of the $X_{F}$ distribution (but once again, not the absolute level) is well predicted for $\overline{\mathrm{p} N} \rightarrow \mu^{+} \mu^{-} \mathrm{x}$ using the COHS structure functions. This reaction 'tests' the Drell-Yan picture more completely than the $\pi^{-N}$ data shown in the same figure since the $\pi N$ dimuon data as a function of $X_{F}$ must be used to extract the pion structure function. In the case of the $\bar{p} N$ reaction the nucleon structure functions can be obtained independently from deep inelastic lepton scattering. The curves in Fig. 2 are the naive DrellYan prediction using COHS structure functions for the $\bar{p} \mathrm{~N}$ reaction and $\mathrm{NA3}$ pion structure functions for the $\pi^{-} \mathrm{N}$ data. These predictions are multiplied by the E-537 K factors given in Table 1 . These data indicate that the structure functions for the $\vec{p}$ and $\pi^{-}$are roughly

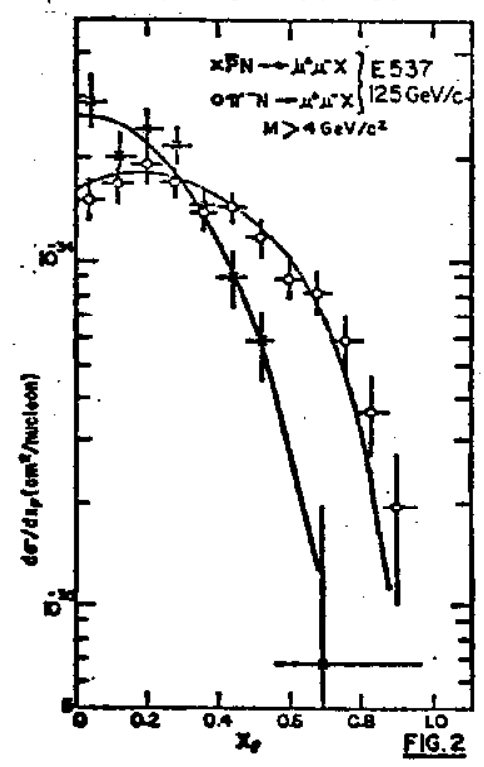


consistent with CDHS and NA3 structure functions respectively. The $\mathrm{E}-537$ experimenters have not yet made an independent determination of structure functions from their data.

Fermilab experiment E-326 has made the independent determination of the pion valence structure function as shown in Fig. 3 using a part of their $225 \mathrm{GeV} / \mathrm{C}^{-} \mathrm{N}$ data. After checking that the factorization of $M^{4} \mathrm{~d} \sigma / d \mathrm{X}_{1} \mathrm{dX}_{2}$ into a product of a function of $x_{1}$ and a function of $x_{2}$ is valid, the E-326 experimenters have extracted the pion structure function $v_{0}\left(x_{1}\right)=(0.75 \pm 0.2) \sqrt{x}(1-x)^{1.01 \pm 0.10}$

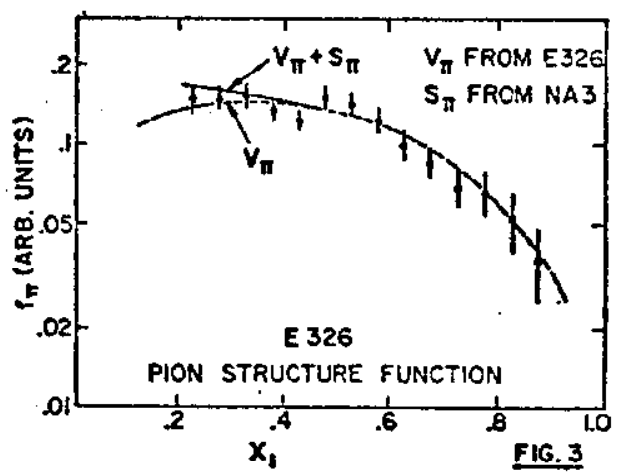
which is consistent with previous NA3 measurement. At the current stage of their analysis, they have used the pion sea quark distributions of NA3 rather than independently determining the sea distribution from their data.

The analysis of the dimuon cross sections as a function of $\sqrt{T}$ and $X_{F}$ that have been, reported above in general depend only on the simple Drell-Yan picture with little or no need for recourse to higher order QCD. However, when the PT spectrum of the dimuon is analyzed, QCD effects appear. Higher order processes in $\alpha_{S}$ such äs gluon Compton scattering or quark-antiquark annihilation contribute and produce high $P_{T}$ muon pairs. Two different techniques have been used in the contributions to the conference to analyze the of the dimuon system. E-537 has analyzed the shape of the $P_{T}$ distributions of their $125 \mathrm{GeV} / \mathrm{C} \overline{\mathrm{p}}$ and $\pi^{-}$data (shown in Fig. 4) by the Altarelli, Parisi, and Petronzio technique. ${ }^{10}$ The nongaussian tail of the $P_{T}$ distribution is explained in this technique by the Compton and annihilation processes. An intrinsic $\left\langle k=220.88 \mathrm{GeV} / \mathrm{c}^{2}\right.$ (which has been observed in $\mathrm{pN}^{\rightarrow} \mu^{+} \mu^{-}$interactions ${ }^{1}$ ), counting rule gluon structure functions and NA3 and CDHS quark structure functions have been used to produce the curves which are shown in Fig. 4. However, this technique requires the assumption that the hard gluon processes are the only contributors to the high $p_{T}$ tail. In fact, soft gluon processes may very well contribute and the $\langle k$ ? $\rangle$ which is needed to explain the data may be an overestimate.

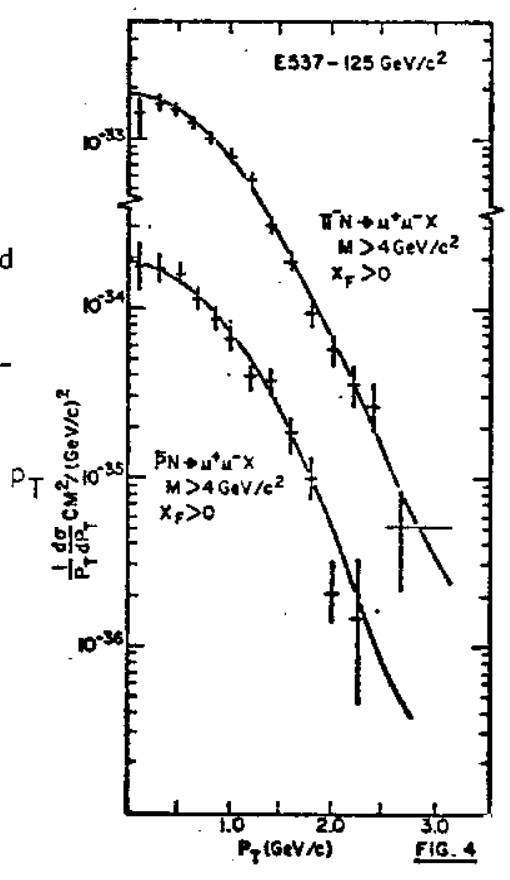

A second method can be used to determine the intrinsic kr of the constituents if the $S$ dependence of $\langle p 2\rangle$ is assumed to be due to QCD processes. Then a linear extrapolation of $\left\langle p \frac{2}{\mathrm{~T}}\right\rangle$ (but not necessarily $\left\langle\mathrm{p}_{\mathrm{T}}\right\rangle$ ) to $S=0$ should yield an 'intrinsic' $\left\langle k_{T}^{2}\right\rangle$. Figure 5 shows $\left\langle p_{T}^{2}\right\rangle$ vs. S for several $\pi^{-} \mathrm{N} \rightarrow$ $\mu^{+} \mu^{-}$measurements? NA3 have fitted their three data points at 150,200 and $280 \mathrm{GeV} / \mathrm{c}$ to a linear form, $\left\langle p \frac{2}{T}\right\rangle=A+B S$. They $f$ ind $\left\langle p_{T}^{2}\right\rangle=(0.76 \pm 0.07)+(0.0025 \pm 0.0002) \mathrm{s} \mathrm{GeV} / \mathrm{c}^{2}$. If all the data in the plot including the new $E-537$ point are fit, $\left\langle p_{T}^{2}\right\rangle=(0.62 \pm 0.05)+$ $(0.0028 \pm 0.0002) \mathrm{s}$ is obtained. This second fit is shown in Fig. 5. Figure 6 shows a similar plot of $\left\langle p \frac{2}{T}\right\rangle$ vs. S prepared for $\mathrm{pN}_{2} \rightarrow \mu^{+} \mu^{-}$. The proton data when fit with $\left\langle p \frac{2}{T}\right\rangle=A+B S$ yields $\left\langle p_{T}^{2}\right\rangle=(0.59 \pm 0.19)+$ $(0.0013 \pm 0.0002) \mathrm{S}$. The slope is less than that observed in Fig. 5, but the intercept which we are defining to be intrinsic $k_{T}$

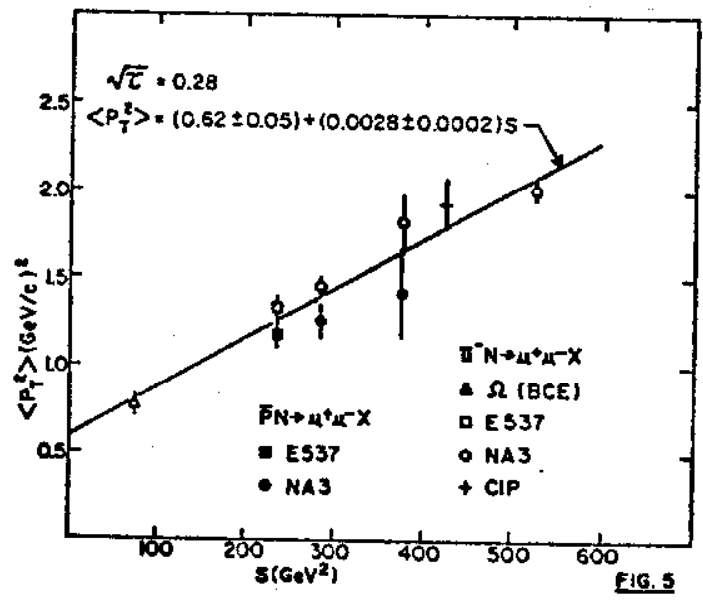


is approximately the same for the proton and pion data. (The three E-288 data points have been lowered slightly to account for the fact that they are $X_{F}=0$ data. The average of all events at $X_{F}>0$ is expected by the Altareli $i$ model to have a $15 \%$ lower $\left\langle p \frac{2}{T}\right\rangle$ than the $X_{F}=0$ data.) In addition the $\bar{p} N$ data (see Fig. 5) from E-537 and NA3 while not of the quality of the $\pi^{-N}$ data seem: to be similar to the pion measurements except that the $\langle p ?\rangle$ for the $\bar{p}$ data is slightly lower at

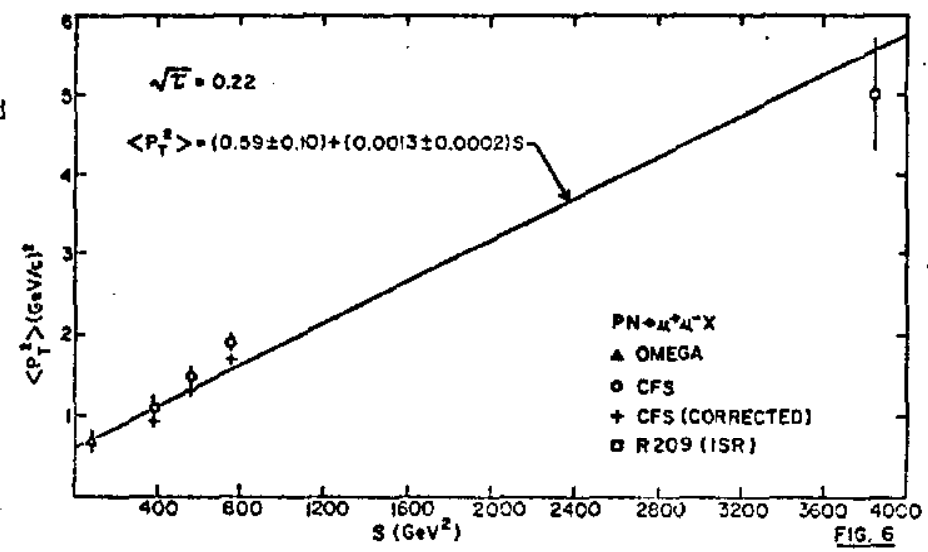
a given energy. Part of the difference in slope of the $S$ dependence may be due to the different $S$ dependence of valencevalence quark interactions (which dominate $\bar{p} N$ and $\pi^{-} N$ reactions) and valence-sea quark interactions (which dominate pN interactions). The observation of similar intercepts of these two plots suggests similar intrinsic $k_{T}$ for valence and sea quarks in protons and pions.

The NA3 experimenters have attempted to predict the slope of their $\pi^{-N}$ data (Fig. 5) due to the annihilation and Compton processes. The expected $\left\langle p \frac{2}{T}\right\rangle$ is given by

$$
\left\langle p_{T}^{2}\right\rangle=\frac{L \int d p_{T}^{2}\left[\frac{d \sigma_{A}}{d p_{T}^{2}}+\frac{d \sigma_{C}}{d p_{T}^{2}}\right] p_{T}^{2}}{k \sigma_{0}}
$$

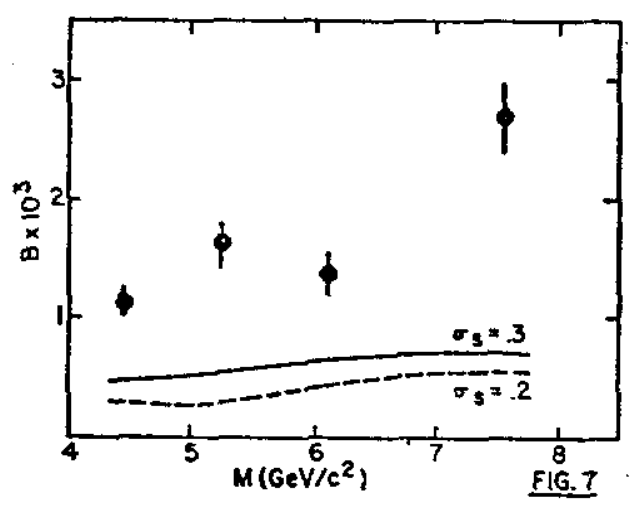

where $\sigma_{A}, \sigma_{C}$, and $\sigma_{0}$ are the annihilation, Compton, and Drell-Yan cross sections respectively. $K$ is the $K$ factor previously defined and $L$ is a corrective multiplier which is used to parameterize the discrepancy between the predicted and observed $\left\langle p_{T}^{2}\right\rangle$. As can be seen from Fig. 7, the calculation (for $L=1, K=1.8$ ) of the slope $\left.\langle p\}\right\rangle$ vs. S falls far short of the observed slope at different masses. For $\alpha_{S}=0.3$, $L / K=1.52 \pm 0.13$ and $L / K$ is increasing with $M$ :

The NA3 experimenters have also displayed $\left\langle p_{T}^{2}\right\rangle$ as a function of $\sqrt{\tau}, y$, and mass at each of their three energies. As seen in Fig. 8a, b, $c$ there are strong variations of $\left\langle p \frac{2}{2}\right\rangle$ with each of these variables and in general the strength of the variation increases with energy. Explanation of this data by quantum chromodynamics will be a challenge for the future.
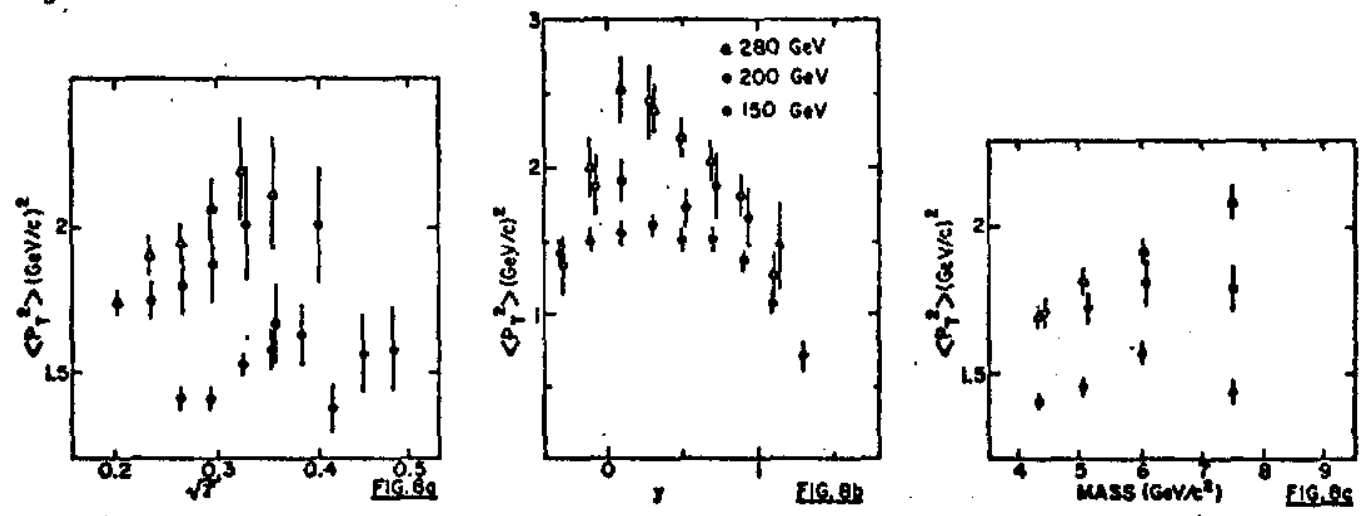
Production of the $\mathrm{J} / \psi$ Resonance - Three new contributions on the production of $\mathrm{J} / \psi$ resonance have been reported at this conference by the E-537, ${ }^{6} \mathrm{NA}^{7}$ and WAI ${ }^{8}$ experimenters. E-537 has studied the antiproton production of $\mathrm{J} / \psi$ at $125 \mathrm{GeV} / \mathrm{c}$ and in particular emphasized the comparison of these cross sections with $\pi^{-N}$ production of $\mathrm{J} / \psi$ at the same energy. The ratio of total cross sections $\left(\sigma-/ \sigma_{\pi}=0.88 \pm 0.05\right)$ shown in Fig. 9a requires a large gluon fusion contribution to $\mathrm{J} 9 \psi \mathrm{p}$ production according to the gluon/quark fusion model of Barger, et al., 12 to equalize the $\vec{p}$ and $\pi^{-}$cross sections. The ratio $\sigma_{p} / \sigma_{-}$shown in $\mathrm{Fig}$. $9 \mathrm{~b}$ indicates that gluon fusion is the most important contribution to $\mathrm{J} / \psi$ production in nucleon-nucleon interactions at high energies, and is needed to explain the energy dependence of the $\sigma / \sigma-$ ratio. The dotted curve in Fig. $9 b$ is the prediction of the $x$ production model $\mathrm{g}_{\mathrm{f}} \mathrm{Carlson}$ and Suaya ${ }^{13}$ in which the $J / \psi^{\prime}$ 's are prociuced through an intermediate $P$ wave state.
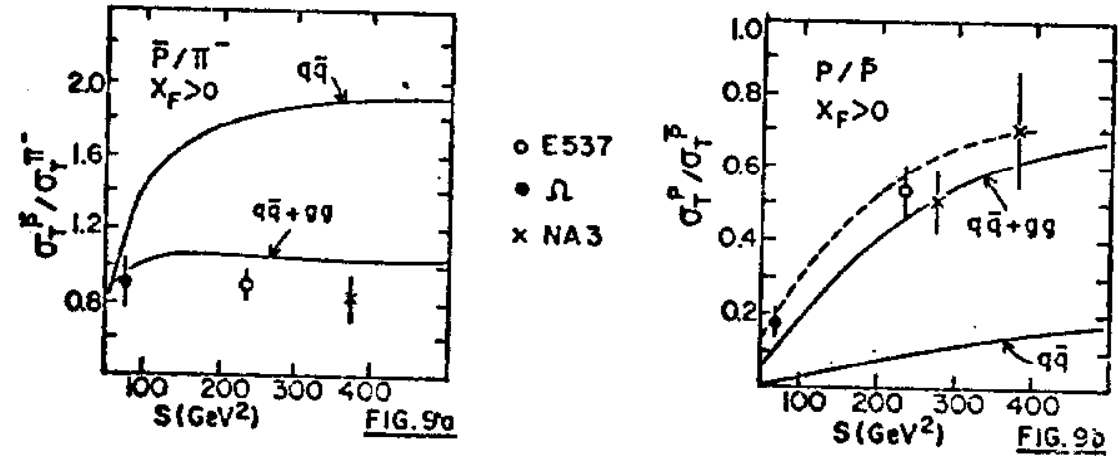

CERN experiment WAll has at tempted to determine the gluon structure function of the pion by analyzing $\mathrm{J} / \psi$ production in their $190 \mathrm{GeV} / \mathrm{C} \pi^{-}$data. In performing this analysis they allowed for a $30.5 \%$ production of $\mathrm{J} / \psi$ by the direct production of a $X$ state which subsequent decays into a $J / \psi$ plus a photon. This corrected $X$ distribution which is shown in $F i g .10$ was fit by varying the input $\pi^{-}$and nucleon structure function to the gluon fusion model for $J / \psi$ production. The results obtained for the pion and nucleon gluon structure functions are $g_{\pi}\left(x_{1}\right) \sim\left(1-x_{1}\right)^{1.9 \pm 0.3}$ and $g_{N}\left(x_{2}\right)=$ $\left(1-x_{2}\right)^{5.5}$ Negligible changes in the pion exponent takes place when the CDHS parameterization is used for the nucleon.

The final new contribution to the conference was the report of NA3 of the detection of $\mathrm{di}-\psi$ production in their $150 \mathrm{GeV} / \mathrm{c}$ and $280 \mathrm{GeV} / \mathrm{c}$ $\pi^{-} N$ data. Out of a sample of $1,359,000 \psi$ events accumulated at the two energies, a total of 13 events with two J/ $\psi^{\prime}$ 's have been found $(6$ at $150 \mathrm{Gev} / \mathrm{c}$ and 7 at $280 \mathrm{GeV} / \mathrm{c}$ ). This yield corresponds to a ratio (after correction for the $\mu^{+} \mu^{-}$branching ratio) of $\sigma \psi \psi / \sigma=(3.0 \pm 1.0) \times 10^{-4}$ and cross sections, $\sigma \psi \psi={ }^{\psi}{ }_{18} \pm 8$ picobarns at $150 \mathrm{GeV} / \mathrm{c}$, $30 \pm 10$ picobarns at $280 \mathrm{GeV} / \mathrm{c}$. The acceptances used to arrive at these cross sections were calculated using either an uncorrelated $\psi \psi$ production or a model which assumes that the $\psi^{\prime}$ 's are decay products of $B$ mesons. The error in the cross sections includes the uncertainty in this acceptance due to the uncertainty in the production process.

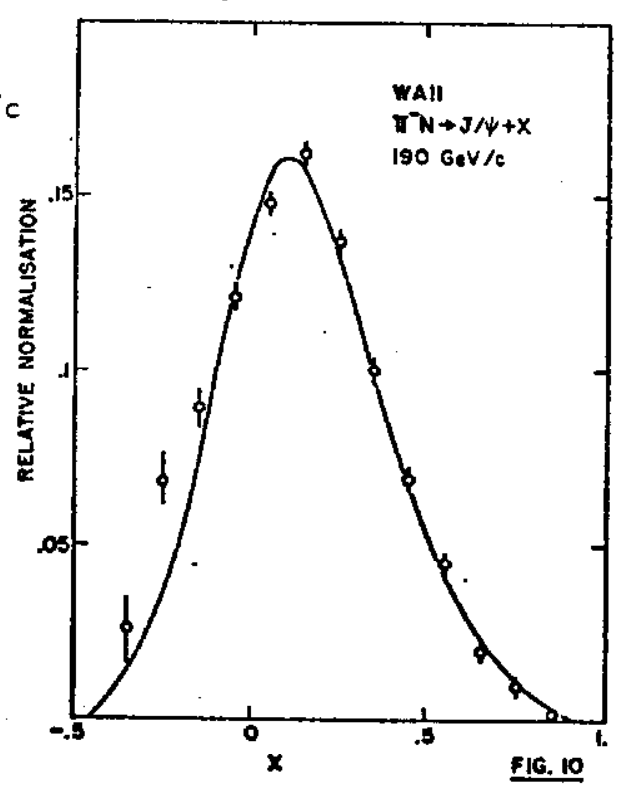




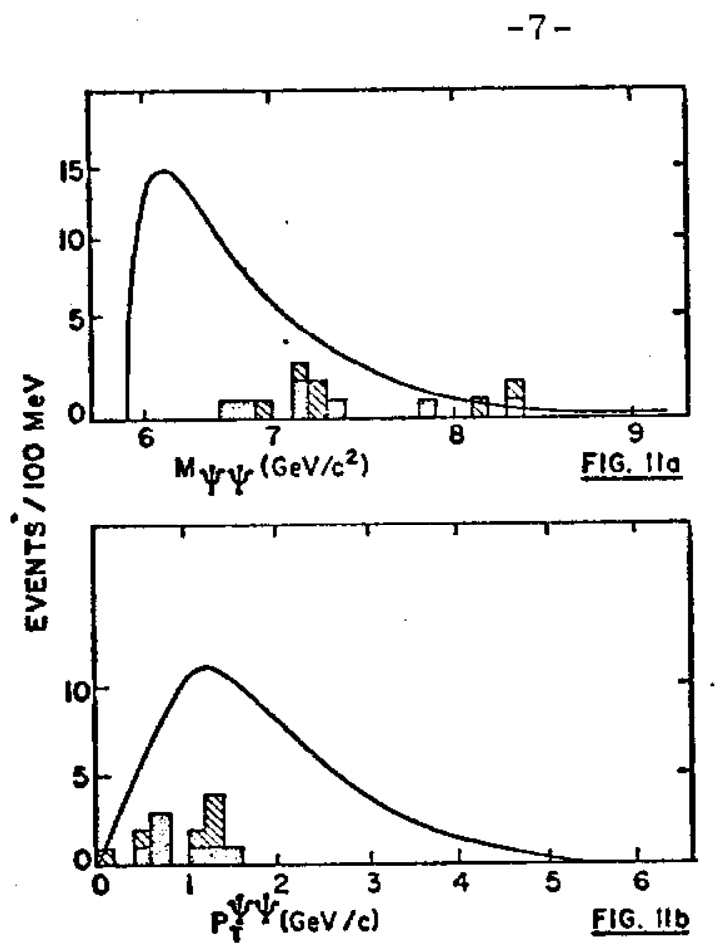

The strong indication as seen in the mass spectrum of the di- $\psi^{\prime} s$ shown in Fig. lla is that the $\psi$ 's are correlated. The curve shown in this figure is the prediction for uncorrelated di-W's. It peaks considerably below the average mass of the observed di- $\psi$ events. (The cross hatched squares are the $150 \mathrm{GeV} / \mathrm{c}$ data.) A second evidence for a correlation between the two $\psi$ 's is the $p_{T}$ spectrum of the di- $\psi$ system shown in Fig. llb. The uncorrelated $\psi$ model prediction peaks somewhat higher than the observed events. The experimenters have made no statement at this time about the production mechanism for the di- $\psi$ 's because of the limited statistics.

Conclusion - Hadronic production of dimuons, both continuum and resonance, continues to be a rich source of information about the constituents of hadrons and their interactions. In particular with the present state of the continuum high mass data, quantum chromodynamics is beginning to be confronted with a wide variety of effects that the theory must explain. The first successes of low order QCD must now be followed with higher order calculations in order to explain the $K$ factor and $\left\langle p_{T}^{2}\right\rangle$ behavior as a function of the various kinetic quantities. The resonance data on the other hand, while not having as complete theoretical framework for interpretation, is beginning to yield information about the gluon composition of the hadrons. In the future we can anticipate both new experiments and new analyses and perhaps new understandings of these fundamental processes.

\section{REFERENCES}

1. DRELL, S.D., and YAN, T.M., Phys. Rev. Letters 25, 316 (1970).

2. HALZEN, F., and SCOTT, D.H., Phys. Rev., D18 (1978), 3778.

3. ANASSONTZIS, E., et al., E-537 Collaboration, paper 665, XXI Int. Conf. on High Energy Physics, Paris, France, 1982/Fermilab Preprint, Conf-82/50-EXP, 7530.537.

4. BADIER, J., et al., NA3 Collaboration, Paper 350, XXI int. Conf. on High Energy Physics, Paris, France, 1982/Eole Polytechnique Preprint LPNE/X 82/02.

5. STICKLAND, D.P., et al., E-326 Collaboration, paper 169, $x \times 1$ Int. Conf. on High Energy Physics, Paris, France, 1982.

6. ANASSONTZIS, E., et al., E-537 Collaboration, paper 656, XXI Int. Conf. on High Energy Physics, Paris, France, 1982/Fermi lab Preprint, Conf-82/49-EXP, 7550.537.

7. PIETRZKY, B., et al., WAll Collaboration, paper 625, XXI Int. Conf. on High Energy Physics, Paris, France, 1982.

8. BADIER, J., NA3 Collaboration, paper 535, XXI Int. Conf. on High Energy Physics, Paris, France, 1982/CERN Preprint, CERN-EP/82-67.

9. DE GROOT, J.G.H., et al., COHS Collaboration, Phys. Letters 82B, 456 (1979).

10. ALTERELLI, et al., Physics Letters, 768, 356 (1978).

11. ITO, A.S., et al., Phys. Rev. D23, 604 (1981).

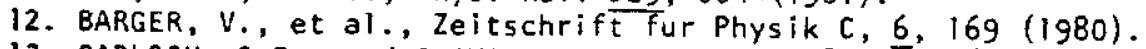

13. CARLSON, C.E., and SUAYA, R., Phys. Rev. 180, 760 (1978). 